\title{
Screening for trisomies by cfDNA testing of maternal blood in twin pregnancy: update of the Fetal Medicine Foundation results and meta-analysis.
}

María del Mar Gil, ${ }^{1,2}$ Slavyana Galeva, ${ }^{1,3}$ Jacques Jani, ${ }^{4}$ Liountmila Konstantinidou, ${ }^{1}$ Ranjit Akolekar, ${ }^{3,5}$ María Nieves Plana, ${ }^{6}$ Kypros H. Nicolaides. ${ }^{1}$

1. Harris Birthright Research Centre of Fetal Medicine, King's College Hospital, London, UK.

2. Universidad Francisco de Vitoria, UFV, Pozuelo de Alarcón, Madrid, Spain.

3. Department of Fetal Medicine, Medway Maritime Hospital, Kent.

4. Department of Obstetrics and Gynecology, University Hospital Brugmann, Brussels, Belgium.

5. Institute of Medical Sciences, Canterbury Christ Church University, Chatham, UK.

6. Department of Preventive Medicine and Public Health, Hospital Príncipe de Asturias, Alcalá de Henares, Madrid. CIBER Epidemiology and Public Health (CIBERESP), Madrid, Spain.

Short title: cfDNA testing in twin pregnancies

Acknowledgement: The study was supported by a grant from The Fetal Medicine Foundation (UK Charity No: 1037116). The cost of collection and analysis of some of the samples for the cell-free DNA test was covered by Roche/Ariosa Diagnostics, Inc. San Jose, CA, USA. These organizations had no role in study design, data collection, data analysis, data interpretation, or writing of the report.

\author{
Corresponding author \\ Professor K Nicolaides \\ Fetal Medicine Research Institute, \\ King's College Hospital, \\ 16-20 Windsor Walk, \\ Denmark Hill, London SE58BB \\ Telephone: +442032998256 \\ Fax: +442077339534
}




\section{ABSTRACT}

Objective: To report on the routine clinical implementation of cell-free (cf)DNA analysis of maternal blood for trisomies 21, 18 and 13 in twin pregnancies and to define the performance of the test by combining our results with those arising from systematic review of the literature.

Methods: The data for the study were derived from prospective screening for trisomies 21,18 and 13 in twin pregnancies at $10^{+0}-14^{+1}$ weeks' gestation. Two populations were included; first self-referred women to the Fetal Medicine Centre in London or Brugmann University Hospital in Brussels and second, women selected for the cfDNA test after routine first-trimester combined testing in one of two National Health Service hospitals in England. This dataset was used to determine the performance of screening for the three trisomies. Search of Medline, Embase, CENTRAL (The Cochrane Library), ClinicalTrials.gov and ICTRP (World Health Organization) was carried out to identify all peer-reviewed publications on clinical validation or implementation of maternal cfDNA testing for trisomies 21, 18 and 13 in twin pregnancies. Meta-analysis was then performed using our data and data from the studies identified by the literature search.

Results: In our dataset of 997 twin pregnancies with a cfDNA result and known outcome, the test classified correctly $16(94.1 \%)$ of the 17 cases of trisomy $21,9(90.0 \%)$ of 10 of trisomy $18,1(50.0 \%)$ of 2 of trisomy 13 and $963(99.5 \%)$ of 968 cases without any of the three trisomies. The literature search identified 7 relevant studies, excluding our papers because their data are included in the current study. In the combined total of our study and the 7 studies identified by the literature search there were 56 trisomy 21 and 3,718 non-trisomy 21 twin pregnancies; the pooled weighted detection rate (DR) and false positive rate (FPR) were 98.2\% (95\% Cl 83.2, 99.8\%) and $0.05 \%(95 \% \mathrm{Cl} 0.01$, $0.26 \%$ ), respectively. In the combined total of 18 cases of trisomy 18 and 3,143 non-trisomy 18 pregnancies the pooled weighted DR and FPR were $88.9 \%(95 \% \mathrm{Cl} 64.8,97.2 \%)$ and $0.03 \%(95 \% \mathrm{Cl}$ $0.00,0.33 \%)$, respectively. For trisomy 13 , there were only 3 affected cases and $2(66.7 \%)$ of these were detected by the cfDNA test at FPR of $0.19 \%(5 / 2,569)$.

Conclusions: Performance of cfDNA testing for trisomies 21 in twin pregnancies is similar to that reported for singleton pregnancies. The number of cases of trisomies 18 and 13 is too small for accurate assessment of predictive performance of the cfDNA test.

KEYWORDS: cell-free DNA; first-trimester screening; non-invasive prenatal testing; trisomy 21; trisomy 18; trisomy 13; twin pregnancy; meta-analysis. 


\section{INTRODUCTION}

In singleton pregnancies, cell-free (cf) DNA analysis of maternal blood provides effective screening for trisomies 21, 18 and 13. A recent meta-analysis of clinical validation studies reported that in the combined total of 1963 cases of trisomy 21 and 223932 non-trisomy 21 singleton pregnancies, the weighted pooled detection rate (DR) was $99.7 \%(95 \% \mathrm{Cl}, 99.1-99.9 \%)$ and false positive rate (FPR) was $0.04 \%(95 \% \mathrm{Cl}, 0.02-0.07 \%)$; in a total of 560 cases of trisomy 18 and 212,019 unaffected pregnancies the pooled weighted DR and FPR were $98.2 \%(95 \% \mathrm{Cl} 95.5-99.2 \%)$ and $0.05 \%(95 \% \mathrm{Cl}$ $0.03-0.07 \%)$ and in a total of 119 cases of trisomy 13 and 212,883 unaffected singleton pregnancies the pooled weighted DR and FPR were $99.0 \%(95 \% \mathrm{Cl} 65.8-100 \%)$ and $0.04 \%(95 \% \mathrm{Cl} 0.02-0.07 \%){ }^{1}$ In contrast to singleton pregnancies, data of cfDNA testing in twins is very limited; the meta-analysis reported that only five studies had examined twin pregnancies prospectively and in a total of 24 cases of trisomy 21 and 1111 non-trisomy 21 cases the DR was $100 \%$ and FPR was $0 \% .^{1}$

In previous studies we reported our data on cfDNA testing for trisomies in twins. In the first study, which included stored and prospectively collected samples, the cfDNA test correctly classified 11 of 12 cases of trisomy 21,1 of 1 of trisomy 18,1 of 1 of trisomy 13 and all 241 non-trisomic pregnancies. ${ }^{2}$ In the second study, we reported the results of prospective screening in twin pregnancies; the cfDNA test correctly classified 11 of 12 cases of trisomy 21,5 of 5 cases of trisomy 18 and all the 334 nontrisomic pregnancies. ${ }^{3}$ In the third study, we reported performance of cfDNA testing in 417 prospectively examined pregnancies; the cfDNA test correctly identified 8 of 8 cases of trisomy 21, 3 of 4 cases of trisomy 18, 0 of the 1 case of trisomy 13 and 403 (99.8\%) of the 404 non-trisomic pregnancies. ${ }^{4}$ In the fourth study, we performed cfDNA testing in pregnancies identified by the combined test as being at intermediate or high risk for trisomies; the cfDNA test correctly identified 6 of 6 cases of trisomy 21, 2 of 3 cases of trisomy 18 and all 206 non-trisomic pregnancies. ${ }^{5}$

The objectives of this study are first, to report our updated experience on prospective first-trimester screening for trisomies in twins by cfDNA testing and second, to carry out a meta-analysis of all studies of cfDNA testing in twin pregnancies published up to $2^{\text {nd }}$ March 2019.

\section{METHODS}

\section{Update of the Fetal Medicine Foundation results}

\section{$\underline{\text { Study design and participants }}$}

The data for the study were derived from prospective screening for trisomies 21,18 and 13 in twin pregnancies at $10^{+0}-14^{+1}$ weeks' gestation. Two populations were included; first self-referred women to the Fetal Medicine Centre in London, which is a private clinic $^{6}$, or Brugmann University Hospital, which is public hospital in Brussels and second, women selected for the cfDNA test after routine firsttrimester combined testing in one of two National Health Service hospitals in England. ${ }^{5,7}$ The patients were examined between October 2012 and January 2018. The study was approved by the appropriate Ethics Committees (NREC reference 13/LO/0885, NREC reference 19/HRA/0576, CE 2014/5).

We recorded maternal characteristics and medical history, including maternal age, racial origin (White, Black, South Asian, East Asian and mixed), method of conception (natural or assisted conception requiring the use of ovulation drugs or in-vitro fertilization), cigarette smoking during pregnancy (yes or no) and parity (parous or nulliparous if no previous pregnancy at or after 24 weeks' gestation). An ultrasound scan was carried out to determine gestational age from the measurement of the crown- 
rump length ${ }^{8}$ of the larger fetus and chorionicity by examining the junction of the intertwin membrane with the placenta. ${ }^{9}$

Women provided written informed consent and maternal blood $(20 \mathrm{~mL})$ was collected into either CellFree DNA BCT tubes (Streck, Omaha, NE) or Roche Cell-Free DNA Collection Tubes (Roche, Pleasanton, CA). These were shipped via courier to Roche/Ariosa Diagnostics, Inc. (San Jose, CA, USA) where they were processed within 7 days of collection. Targeted cfDNA testing for fetal trisomy was performed using the Harmony ${ }^{\circledR}$ prenatal test as described previously. ${ }^{10-13}$ Harmony® uses DANSR assays targeting sequences on chromosomes 13, 18, and 21 for chromosome quantitation and single nucleotide polymorphisms on chromosomes 1 to 12 for fetal fraction measurement. Products of the DANSR assays can be quantified using either next-generation sequencing or a custom microarray; both were used during the course of this study. The data are analysed with the fetal fraction optimized FORTE algorithm, which calculates probability scores for fetal trisomy, with greater than $1 \%$ considered to be high probability. In cases where the cfDNA test did not provide results the parents were offered repeat testing or to rely on the results of the combined test in deciding whether to have an invasive test or not. In cases with a high-risk result from the cfDNA test, the parents were advised to consider having invasive fetal karyotyping before deciding on the further management of their pregnancy.

Patient characteristics, results of the investigations and pregnancy outcome were recorded in a database. The outcomes were divided into first, trisomy 21,18 or 13 if the karyotype of chorionic villi, amniotic fluid or neonatal blood demonstrated the relevant trisomy in one or both babies, second, no trisomy 21,18 or 13 if the karyotype was normal or both neonates were phenotypically normal, third, no known karyotype in both fetuses because the pregnancies resulted in termination or embryo reduction, miscarriage or stillbirth and no karyotyping of fetal tissue was carried out, and fourth, outcome unknown because the pregnancies were lost to follow up.

$\underline{\text { Statistical analyses }}$

Descriptive data were presented in median and interquartile range (IQR) for continuous variables and in numbers and percentages for categorical variables.

\section{Systematic review and meta-analysis}

\section{Literature search and study selection}

Searches of Medline via PubMed, Embase and CENTRAL (The Cochrane Library) were performed to identify clinical validation or implementation studies of maternal cfDNA testing in screening for aneuploidies in twin pregnancies; additionally, ClinicalTrials.gov and WHO International Clinical Trials Registry Platform (ICTRP) were also searched for ongoing or recently completed trials. The study period was from January 2011 , when the first such study was published, to $9^{\text {th }}$ March 2019 ; the initial search was $8^{\text {th }}$ December 2018 and this was updated with autoalerts in Medline. A list of relevant citations was generated from these databases using the search strategies included in Appendix 1. This review was registered in PROSPERO international database for systematic reviews (reference: CRD42019121506).

The abstracts of citations were examined by two reviewers (M.M.G., S.G) to identify all potentially relevant articles, which were then examined in full-text form. Reference lists of relevant original and review articles were hand-searched for additional reports. Agreement about potential relevance was reached by consensus and by consultation with a third reviewer (K.H.N.). 
The inclusion criteria were peer-reviewed studies reporting on clinical validation or implementation of maternal cfDNA testing in screening for aneuploidies in twin pregnancies, in which data on pregnancy outcome were provided for more than $85 \%$ of the study population. Proof-of-principle articles and studies in which the laboratory scientists carrying out the tests were aware of fetal karyotype or pregnancy outcome were excluded. We also excluded case-control studies because they tend to introduce an optimistic bias to the estimates of diagnostic performance.

\section{Data extraction and meta-analysis of data from all studies}

Data regarding sample size, gestational age at analysis, method used for cfDNA testing and DR or sensitivity and FPR or specificity for non-mosaic trisomies 21,18 and 13 were obtained from each study included in the systematic review and documented in contingency tables. In the construction of these tables, we used the results from the cfDNA test and excluded those cases in which the test failed to give a result. In the calculation of FPR, we included all euploid and aneuploid cases other than the aneuploidy under investigation. Authors were contacted when clarifications were required in the interpretation of the data.

We extracted data from the primary studies to obtain the four cell values of a diagnostic $2 \times 2$ table to calculate test accuracy measures of DR and FPR. The analyses were stratified according to the different conditions (trisomy 21 and trisomy 18). We calculated DR and FPR with corresponding 95\% Cls for individual studies and displayed them in forest plots to investigate heterogeneity. We pooled the DR and FPR estimates using bivariate random-effects regression models. The bivariate model assumes that logit transformations of DR and FPR are negatively correlated and follow a bivariate normal distribution ${ }^{14}$. We computed the positive and negative likelihood ratios from the pooled estimates of DR and FPR. Heterogeneity among studies was quantified with the variance of the logit of accuracy indices as estimated by the bivariate model.

Publication bias was not analyzed given the limited power of available tests and the uncertainty about interpreting statistical evidence of funnel plot asymmetry as necessarily implying publication bias. ${ }^{15}$ For trisomy 13 there was an insufficient number of cases for meaningful meta-analysis and we therefore computed average DR and FPR values.

We conducted statistical analyses using the Metandi and Midas programs for the STATA software. ${ }^{16}$

\section{RESULTS}

\section{Update of the Fetal Medicine Foundation results}

\section{Study population}

A total of 1122 twin pregnancies had cfDNA testing, but $125(11.1 \%)$ of these were excluded from further analysis either because the cfDNA test did not provide a result $(n=52)$, the pregnancies ended in termination, miscarriage or stillbirth with no known karyotype $(n=45)$ or there was loss to follow up $(n=28)$.

In the 997 cases included in the study, 854 (85.7\%) were dichorionic and $143(14.3 \%)$ were monochorionic; the median maternal age was 38.0 (IQR 34.5-41.0) years, the median maternal weight was 69.0 (IQR 60.4-82.6) $\mathrm{kg}$ and the median gestational age at sampling was 12.1 (IQR 10.7-12.9) weeks. The racial origin of the women was White in 772 (77.4\%), South Asian in 65 (6.5\%), East Asian in $32(3.2 \%)$, Black in $104(10.4 \%)$ and mixed in $24(2.4 \%)$. Conception was natural in 766 
(76.8\%) and after use of assisted reproductive techniques in 231 (23.2\%).

The study population of 997 pregnancies included 17 with trisomy 21,10 with trisomy 18,2 with trisomy 13 and 968 without trisomy 21, 18 or 13; one case of trisomy 18 was in a monochorionic twin pregnancy where the two fetuses were affected and all the other trisomic cases were in the dichorionic pregnancies where only one fetus was trisomic and the cotwin was non-trisomic.

\section{Performance of screening}

The cfDNA test classified correctly $16(94.1 \%)$ of the 17 cases of trisomy $21,9(90.0 \%)$ of 10 of trisomy 18, $1(50.0 \%)$ of 2 of trisomy 13 and $963(99.5 \%)$ of 968 cases without any of the three trisomies. One case each of trisomy 21 , trisomy 18 and trisomy 13 was classified as normal. In the non-trisomic group, 4 cases were classified as trisomy 13 and 1 as trisomy 21 and therefore, the combined FPR was $0.52 \%$ (5/968).

\section{Systematic review and meta-analysis}

\section{Data sources}

The search identified 329 potentially relevant citations, but 322 were excluded because they were non-relevant publications, conference abstracts rather than peer-reviewed papers, review articles or opinions, studies not on twins, case-control studies, or studies on clinical implementation of cfDNA testing in screening for aneuploidies in which pregnancy outcome data were provided for fewer than $85 \%$ of the study population, proof-of-principle studies reporting laboratory techniques rather than clinical validation of a predefined method of maternal blood cfDNA analysis (Figure 1). In total, 9 relevant studies were identified ${ }^{4,5,17-23}$ but two of these ${ }^{4,5}$ were excluded from the meta-analysis because their data are included in the updated Fetal Medicine Foundation results presented above. The characteristics of our current study and the seven ones identified by the literature search are summarized in Table 1.

\section{Methodological quality of the selected studies}

The methodological quality of the selected studies, assessed by the Quality Assessment tool for Diagnostic Accuracy Studies (QUADAS-2) ${ }^{24}$ is illustrated in Figure 2. This tool comprises four domains; each one is assessed in terms of risk of bias and the first three are also assessed in terms of concerns regarding applicability.

\section{Risk of bias}

The first domain relates to patient selection. A study was considered to be at low risk of bias if the cfDNA test was carried out in a consecutive or random sample of patients; all the studies were classified as being at high risk of bias because either the samples were not explicitly stated to have been consecutive or selected at random. The second domain relates to the index test; all included studies were considered to be at low risk of bias because the cfDNA test was carried out and the results given by the laboratory without prior knowledge of the fetal karyotype or pregnancy outcome. The third domain relates to the reference standard; all included studies were considered to be at low risk of bias because the method of diagnosing the chromosomal abnormality under investigation including karyotyping or neonatal examination was accepted to be true. The fourth domain relates to flow and timing. A study was considered to be at low risk of bias if first, in the calculation of performance of screening, all patients in the study population had a result from both the cfDNA test and pregnancy outcome and, second, if the method of classifying the outcome result (invasive testing 
or clinical examination) was the same in all cases in the study population. All but one study ${ }^{18}$ were classified as being at high risk of bias because cfDNA testing was not carried out or did not provide results in all cases and/or there was no complete follow-up and/or the method of determining outcome was not the same in all cases.

\section{Concerns regarding applicability}

In the context of screening for fetal aneuploidies by cfDNA analysis of maternal blood in the general population, the first domain relates to patient selection and all the studies were classified as being at high risk of concerns regarding applicability because in these studies the test was not carried out in the general population but in a mixture of low- and high-risk pregnancies, where some of the patients had had another screening test before opting for cfDNA testing. In terms of the second and third domains on index test and reference standard, respectively, all studies were classified as being at low risk of bias and were also considered as being at low risk of concerns regarding applicability.

\section{Method of analyzing samples}

The studies included in the meta-analysis used one of two methods for analysis of cfDNA in maternal blood: massively parallel sequencing or targeted analysis (either by next-generation sequencing or by a custom microarray) (Table 1).

\section{Nature of the studies}

All studies included in this meta-analysis were prospective; three studies were in high-risk pregnancies and five examined a mixture of high-risk and routine populations (Table 1). The proportion of monochorionic twin pregnancies ranged from $3.2 \%$ to $42.4 \%$.

\section{Meta-analysis and performance of screening for aneuploidies}

The DR and FPR for each study, weighted pooled data and heterogeneity between studies (variance of the logit sensitivity and specificity) are provided in Tables 2 and 3; sensitivity and specificity are illustrated in Figures 3 and 4. Heterogeneity between studies was very low (Tables 2 and 3).

\section{Trisomy 21}

A total of 8 studies reported on the performance of screening by cfDNA analysis for trisomy 21 in a combined total of 56 cases of trisomy 21 and 3718 non-trisomy 21 twin pregnancies (Table 2, Figure 3). Among individual studies, the DR varied between $94.1 \%$ and $100 \%$ and the FPR varied between $0 \%$ and $0.24 \%$. The weighted pooled DR and FPR were $98.2 \%(95 \% \mathrm{Cl} 83.2,99.8 \%)$ and $0.05 \%$ $(95 \% \mathrm{Cl} 0.01,0.26 \%)$, respectively.

\section{Trisomy 18}

A total of 5 studies reported on the performance of screening by cfDNA analysis for trisomy 18 in a combined total of 18 cases of trisomy 18 and 3,143 non-trisomy 18 twin pregnancies (Table 3, Figure 4). Among individual studies, the DR varied between $50.0 \%$ and $100 \%$ and the FPR varied between $0 \%$ and $0.10 \%$. The weighted pooled DR and FPR were $88.9 \%(95 \% \mathrm{Cl} 64.8,97.2 \%)$ and $0.03 \%$ $(95 \% \mathrm{Cl} 0.00,0.33 \%)$, respectively.

\section{Trisomy 13}


A total of three studies reported on the performance of screening by cfDNA analysis for trisomy 13 in a combined total of three cases of trisomy 13 and 2,549 non-trisomy 13 twin pregnancies. In our study 1 of 2 affected cases were detected by cfDNA testing at FPR of $0.4 \%$ (4/995). In the second study ${ }^{23}$ the one affected case was detected at FPR of $0 \%(0 / 1156)$. In the third study ${ }^{21}$ there were no cases of trisomy 13 but one false positive result. In the combined results the DR was $66.7 \%(2 / 3)$ and FPR was $0.20(5 / 2,549)$.

\section{DISCUSSION}

\section{Principal findings of the study}

The results of our study and the meta-analysis of cfDNA testing in maternal blood in twin pregnancies suggest that the performance of the test for trisomy 21 may be similar to that in singleton pregnancies. In the combined total of 56 trisomy 21 and 3,718 non-trisomy 21 twin pregnancies the pooled weighted DR and FPR were $98.2 \%(95 \% \mathrm{Cl} 83.2,99.8 \%)$ and $0.05 \%(95 \% \mathrm{Cl} 0.01,0.26 \%)$, respectively; in our meta-analysis of studies in singleton pregnancies the weighted pooled DR in 1,963 cases of trisomy 21 was $99.7 \%(95 \% \mathrm{Cl}, 99.1-99.9 \%)$ and the FPR in 223,932 non-trisomy 21 pregnancies was $0.04 \%(95 \% \mathrm{Cl}, 0.02-0.07 \%) .{ }^{1}$ In the combined total of 18 cases of trisomy 18 and 3,143 non-trisomy 18 pregnancies the pooled weighted DR and FPR were $88.9 \%(95 \% \mathrm{Cl} 64.8$, $97.2 \%)$ and $0.03 \%(95 \% \mathrm{Cl} 0.00,0.33 \%)$, respectively; in our meta-analysis of studies in singleton pregnancies the weighted pooled DR in 563 cases of trisomy 18 was $97.9 \%(95 \% \mathrm{Cl}, 94.9-99.1 \%)$ and the FPR in 222,013 non-trisomy 18 pregnancies was $0.04 \%, 95 \% \mathrm{Cl} 0.03-0.07 \%) .{ }^{1}$ The number of twin pregnancies with trisomy $13(n=3)$ is too small for accurate assessment of DR. The average FPR for trisomy 13 of $0.19 \%(5 / 2,569)$ seems slightly higher than the values reported for singleton pregnancies $(0.04 \%, 95 \% \mathrm{Cl} 0.02-0.07 \%) .{ }^{1}$

In our study the method of cfDNA testing was targeted but in all other studies MPSS was used. Similarly, our study was confined to pregnancies in the first-trimester, whereas the other studies included pregnancies in the second and some in the third trimester. Although there was no obvious difference in performance of screening between our and that of the other studies the small number of cases prevented meaningful subgroup analyses, including chorionicity, cfDNA method for analysis, patient's background risk or gestational age at testing.

This study has not addressed the issue of cfDNA test failure because we have recently reported our experience in a larger cohort including both singleton and twin pregnancies. ${ }^{25}$ In that study we found that important contributors to cfDNA test failure are increased maternal weight, conception by in vitro fertilization, Black or South Asian racial origin, dichorionicity, nulliparity, and low gestational age and low serum PAPP-A and free B-hCG. Test failure after first sampling in dichorionic twins was 3.3 times higher than in singletons but to a great extent this excess failure rate can be attributed to the fact that a considerably higher proportion of twins were conceived by in vitro fertilization and more women were nulliparous.

\section{Comparison with results of previous meta-analyses in twin pregnancies}

Our previous meta-analysis on the performance of cfDNA testing for fetal aneuploidies in clinical validation or implementation studies included five studies in twin pregnancies; in a total of 24 cases of trisomy 21 and 1,111 unaffected cases the DR was $100 \%$ and FPR was $0 \% .{ }^{1}$ Another meta-analysis examined four studies that included both singletons and twins and three studies that included only twins; it was not possible to extract the number of twin pregnancies that were evaluated but the 
authors reported that the DR of trisomy 21 in twins was 89.4\% (95\% 75-96\%) and the FPR was $0.4 \% .^{26}$

Liao et al., conducted a meta-analysis of studies reporting on cfDNA testing in twin pregnancies. ${ }^{27}$ They included 10 studies, one of which was retrospective, three were a mixture of retrospective and prospective and six were prospective; five were cohort studies, two were case-control studies and three had a mixture of cohort and case-control studies. The authors did not set any criteria on degree of follow up. In a combined total of 69 cases of trisomy 21 the DR was $99 \%(95 \% \mathrm{Cl}, 92-100 \%)$, in 13 cases of trisomy 18 the DR was $85 \%(95 \% \mathrm{Cl}, 55-98 \%)$, in 3 cases of trisomy 13 the DR was $100 \%$ and in 2008 euploid pregnancies the FPR was $0.05 \%$. Our meta-analysis included only prospective cohort studies with follow-up in at least $85 \%$ of cases to avoid reporting bias. Case-control studies were excluded because they tend to introduce an optimistic bias to the estimates of diagnostic performance.

\section{Implication for clinical practice}

This meta-analysis provides good evidence that the performance of cfDNA testing for trisomy 21 in twin pregnancies may be similar to that in singletons. In this respect, the performance of the cfDNA test is superior, both in terms of higher DR and substantially lower FPR, to that of the first trimester combined test or second trimester biochemical testing. ${ }^{28}$ This is particularly important in the case of dichorionic twins where both the incidence of aneuploidies and the invasive procedure-related risk of pregnancy loss are increased compared to singletons. If the pregnancies are discordant for an aneuploidy and the parents choose to have selective fetocide, the subsequent risk of miscarriage or early preterm birth increase with gestational age at fetocide; ${ }^{29}$ in this respect it would be preferable to offer screening leading to prenatal diagnosis in the first than the second trimester.

A positive or high-risk cfDNA result should be confirmed by invasive testing. In the case of firsttrimester screening and positive cfDNA result for trisomy 21 the diagnostic test can be chorionic villous sampling. In the case of trisomies 18 and 13 a positive result should be followed by a detailed ultrasound examination and if the characteristic defects associated with these trisomies are detected then chorionic villous sampling can be carried out; if no defects are detected in the scan the preferred diagnostic test is amniocentesis to avoid an erroneous result due to placenta confined mosaicism.

On the other hand, a negative or low-risk cfDNA result is reassuring that the fetus is unlikely to be affected by the trisomy under investigation. The posterior risk for a given patient can be obtained by multiplying the prior risk with the negative likelihood ratios calculated in this meta-analysis; the risk for trisomies 21 and 18 is reduced by a factor of 56 and 9, respectively. For example, if prior screening by the combined test had shown that the risk for trisomy 21 was 1 in 10 and cfDNA testing gives a lowrisk result the chance that the fetus is affected is 1 in 560; in contrast, if the risk for trisomy 18 from the combined test was 1 in 2 and cfDNA testing gives a low-risk result the chance that the fetus is affected is 1 in 18 .

\section{Limitations}

Contrary to our previous meta-analysis in singleton pregnancies, the number of published studies analysing performance of cfDNA testing in twin pregnancies is very limited and consequently, the number of affected cases included in this meta-analysis is considerably smaller. However, the results reported in the literature for trisomies 21 and 18 present low heterogeneity and therefore they are likely to represent the real performance. There is an insufficient number of trisomy 13 cases to accurate assess performance. 
In the assessment of the quality of the included studies, all of them were considered to be at high-risk of selection bias and at high-risk of concerns regarding applicability in relation to patient selection because they were not performed as part of routine primary screening but they were carried out in preselected populations. However, the ability to detect aneuploidy with cfDNA analysis is dependent upon assay precision and fetal DNA percentage in the sample rather than the prevalence of the disease in the study population. Most studies were also classified as being at high-risk of bias in relation to flow and timing. This is essentially because cfDNA testing did not provide results in all cases, there was no complete follow up, or the method of determining outcome was not the same in all cases. However, such criticisms could be applied to any clinical study; all methods of traditional screening occasionally fail to give a result and no screening study in pregnancy can have complete follow up, especially because some women miscarry and karyotyping is not carried out.

\section{Conclusions}

Performance of cfDNA testing for trisomy 21 in twin pregnancies may be similar to that reported for singleton pregnancies. The number of cases of trisomies 18 and 13 is too small for accurate assessment of predictive performance of the cfDNA test. 


\section{REFERENCES}

1. Gil MM, Accurti V, Santacruz B, Plana MN, Nicolaides KH. Analysis of cell-free DNA in maternal blood in screening for aneuploidies: updated meta-analysis. Ultrasound Obstet Gynecol 2017; 50: 302-314.

2. Del Mar Gil M, Quezada MS, Bregant B, Syngelaki A, Nicolaides KH. Cell-free DNA analysis for trisomy risk assessment in first-trimester twin pregnancies. Fetal Diagn Ther 2014; 35: 204-211.

3. Bevilacqua E, Gil MM, Nicolaides KH, Ordoñez E, Cirigliano V, Dierickx H, Willems, PJ, Jani JC. Performance of screening for aneuploidies by cell-free DNA analysis of maternal blood in twin pregnancies. Ultrasound Obstet Gynecol 2015 Jan; 45: 61-66.

4. Sarno L, Revello R, Hanson E, Akolekar R, Nicolaides KH. Prospective first-trimester screening for trisomies by cell-free DNA testing of maternal blood in twin pregnancy. Ultrasound Obstet Gynecol 2016; 47: 705-711.

5. Galeva S, Konstantinidou L, Gil MM, Akolekar R, Nicolaides KH. Routine first-trimester screening for fetal trisomies in twin pregnancies: cell-free DNA test contingent on results from the combined test. Ultrasound Obstet Gynecol 2019; 53: 208-213.

6. Quezada MS, Gil MM, Francisco C, Oròsz G, Nicolaides KH. Screening for trisomies 21, 18 and 13 cell-free DNA analysis of maternal blood at 10-11 weeks' gestation and the combined test at 11-13 weeks. Ultrasound Obstet Gynecol 2015; 45: 36-41.

7. Gil MM, Revello R, Poon LC, Akolekar R, Nicolaides KH. Clinical implementation of routine screening for fetal trisomies in the UK NHS: cell-free DNA test contingent on results from firsttrimester combined test. Ultrasound Obstet Gynecol 2016; 47: 45-52.

8. Robinson HP, Fleming JE. A critical evaluation of sonar crown rump length measurements. $\mathrm{Br}$ J Obstet Gynaecol 1975; 82: 702-710.

9. Sepulveda W, Sebire NJ, Hughes K, Odibo A, Nicolaides KH. The lambda sign at 10-14 weeks of gestation as a predictor of chorionicity in twin pregnancies. Ultrasound Obstet Gynecol 1996; 7: 421-423.

10. Sparks AB, Struble CA, Wang ET, Song K, Oliphant A. Noninvasive prenatal detection and selective analysis of cell-free DNA obtained from maternal blood: evaluation for trisomy 21 and trisomy 18. Am J Obstet Gynecol 2012; 206: 319.e1-9.

11. Ashoor G, Syngelaki A, Wang E, Struble C, Oliphant A, Song K, Nicolaides KH. Trisomy 13 detection in the first trimester of pregnancy using a chromosome-selective cell-freeDNAanalysis. Ultrasound Obstet Gynecol 2013; 41: 21-25.

12. Struble CA, Syngelaki A, Oliphant A, Song K, Nicolaides $\mathrm{KH}$. Fetal fraction estimate in twin pregnancies using directed cell-free DNA analysis. Fetal Diagn Ther 2014; 35: 199-203.

13. Juneau K, Bogard PE, Huang S, Mohseni M, Wang ET, Ryvkin P, Kingsley C, Struble CA, Oliphant A, Zahn JM. Microarray-based cell-free DNA analysis improves noninvasive prenatal testing. Fetal Diagn Ther 2014; 36: 282-286. 
14. Reitsma JB, Glas AS, Rutjes AW, Scholten RJ, Bossuyt PM, Zwinderman AH. Bivariate analysis of sensitivity and specificity produces informative summary measures in diagnostic reviews. J Clin Epidemiol 2005; 58: 982-990.

15. Leeflang MM, Deeks JJ, Gatsonis C, Bossuyt PM, Cochrane Diagnostic Test Accuracy Working Group. Systematic reviews of diagnostic test accuracy. Ann Intern Med 2008; 149: 889-897

16. StataCorp. 2015. Stata Statistical Software: Release 14. College Station, TX, USA)

17. Lau TK, Jiang F, Chan MK, Zhang H, Lo PS, Wang W. Non-invasive prenatal screening of fetal Down syndrome by maternal plasma DNA sequencing in twin pregnancies. $J$ Matern Fetal Neonatal Med 2013; 26: 434-437.

18. Huang X, Zheng J, Chen M, Zhao Y, Zhang C, Liu L, Xie W, Shi S, Wei Y, Lei D, Xu C, Wu Q, Guo X, Shi X, Zhou Y, Liu Q, Gao Y, Jiang F, Zhang H, Su F, Ge H, Li X, Pan X, Chen S, Chen F, Fang $Q$, Jiang $H$, Lau TK, Wang W. Noninvasive prenatal testing of trisomies 21 and 18 by massively parallel sequencing of maternal plasma DNA in twin pregnancies. Prenat Diagn 2014; 34: 335-340.

19. Tan Y, Gao Y, Lin G, Fu M, Li X, Yin X, Du J, Li J, Li W, Peng H, Yuan Y, Chen F, Jiang F, Zhang H, Lu G, Gong F, Wang W. Noninvasive prenatal testing (NIPT) in twin pregnancies with treatment of assisted reproductive techniques (ART) in a single center. Prenat Diagn 2016; 36: 672679.

20. Du E, Feng C, Cao Y, Yao Y, Lu J, Zhang Y. Massively Parallel Sequencing (MPS) of Cell-Free Fetal DNA (cffDNA) for Trisomies 21, 18, and 13 in twin pregnancies. Twin Res Hum Genet 2017; 20: 242-249.

21. Le Conte G, Letourneau A, Jani J, Kleinfinger P, Lohmann L, Costa JM, Benachi A. Cell-free fetal DNA analysis in maternal plasma as screening test for trisomies 21, 18 and 13 in twin pregnancy. Ultrasound Obstet Gynecol 2018; 52: 318-324.

22. Yang J, Qi Y, Hou Y, Guo F, Peng H, Wang D, Haoxin OY, Wang Y, Huang H, Yin A. Performance of non-invasive prenatal testing for trisomies 21 and 18 in twin pregnancies. Mol Cytogenet 2018; 11: 4-9.

23. Yu W, Lv Y, Yin S, Liu H, Li X, Liang B, Kong L, Liu C. Screening of fetal chromosomal aneuploidy diseases using noninvasive prenatal testing in twin pregnancies. Expert Rev Mol Diagn 2018; doi: 10.1080/14737159.2019.1562906.

24. Whiting PF, Rutjes AW, Westwood ME, Mallett S, Deeks JJ, Reitsma JB, Leeflang MM, Sterne JA, Bossuyt PM; QUADAS-2 Group. QUADAS-2: a revised tool for the quality assessment of diagnostic accuracy studies. Ann Intern Med 2011; 155: 529-536.

25. Galeva S, Gil MM, Konstantinidou L, Akolekar R, Nicolaides KH. First-trimester screening for trisomies by cfDNA testing of maternal blood in singleton and twin pregnancies: factors affecting test failure. Ultrasound Obstet Gynecol, in press.

26. Taylor-Phillips S, Freeman K, Geppert J, Agbebiyi A, Uthman OA, Madan J, Clarke A, Quenby $S$, Clarke A. Accuracy of non-invasive prenatal testing using cell-free DNA for detection of Down, Edwards and Patau syndromes: a systematic review and meta-analysis. BMJ Open 2016; 6: e010002. 
27. Liao H, Liu S, Wang H. Performance of non-invasive prenatal screening for fetal aneuploidy in twin pregnancies: a meta-analysis. Prenat Diagn. 2017; 37: 874-882.

28. Audibert F, Gagnon, A. Prenatal screening for and diagnosis of aneuploidy in twin pregnancies. J Obstet Gynaecol Canada 2017;39:e329-46.

29. Evans MI, Goldberg JD, Dommergues M, Wapner RJ, Lynch L, et al. Efficacy of secondtrimester selective termination for fetal abnormalities: International collaborative experience among the world's largest centers. Am J Obstet Gynecol 1994; 171: 90-94. 


\section{FIGURE LEGENDS}

Figure 1. Flow chart for the systematic review.

Figure 2. Summary of the quality of included studies using the Quality Assessment tool for Diagnostic Accuracy Studies (QUADAS-2) checklist.

Figure 3. Forest plots of sensitivity and specificity with $95 \%$ confidence intervals $(\mathrm{Cl})$ and weighted pooled summary statistics using bivariate random-effects model in assessing cell-free DNA analysis in screening for trisomy 21.

Figure 4. Forest plots of sensitivity and specificity with $95 \%$ confidence intervals $(\mathrm{Cl})$ and weighted pooled summary statistics using bivariate random-effects model in assessing cell-free DNA analysis in screening for trisomy 18. 
Table 1. Summary of characteristics of studies reporting on cell-free (cf) DNA analysis of maternal blood in screening for trisomies (T) 21, 18 and 13 in twin pregnancies.

\begin{tabular}{|c|c|c|c|c|c|c|c|c|c|c|}
\hline Study & $\begin{array}{l}\text { Aneuploidy } \\
\text { included }\end{array}$ & $\mathbf{N}$ & Monochorionic & $\begin{array}{l}\text { T21 } \\
\text { (n) }\end{array}$ & $\begin{array}{l}\text { T18 } \\
\text { (n) }\end{array}$ & $\begin{array}{l}\text { T13 } \\
\text { (n) }\end{array}$ & $\begin{array}{c}\text { Outcome } \\
\text { known } \\
(\%)\end{array}$ & $\begin{array}{l}\text { cfDNA } \\
\text { method }\end{array}$ & GA (weeks) & Population \\
\hline Lau $(2013)^{17}$ & T21 & 12 & $2(16.7 \%)$ & 1 & & & 100 & MPSS & $13(11-20)$ & High-risk \\
\hline Huang $(2014)^{18}$ & T21, T18 & 189 & $33(17.5 \%)$ & 9 & 2 & & 100 & MPSS & $19(11-36)$ & High-risk \\
\hline $\operatorname{Tan}(2016)^{19}$ & T21 & 510 & $3.2 \% *$ & 4 & & & 90 & MPSS & $12(11-28)$ & Mixture \\
\hline Du $(2017)^{20}$ & T21 & 92 & $39(42.4 \%)$ & 2 & & & 100 & MPSS & $18(14-23)$ & High-risk \\
\hline Le Conte $(2018)^{21}$ & T21, T18, T13 & 418 & $86(20.6 \%)$ & 3 & 1 & & 85 & MPSS & $16(10-35)$ & Mixture \\
\hline Yang $(2018)^{22}$ & T21, T18 & 432 & $95(22.0 \%)$ & 4 & 1 & & 91 & MPSS & $>9$ & Mixture \\
\hline Yu $(2019)^{23}$ & T21, T18, T13 & 1157 & $308(26.6 \%)$ & 16 & 4 & 1 & 99 & MPSS & $18(8-30)$ & Mixture \\
\hline Current study & T21, T18, T13 & 997 & $143(14.3 \%)$ & 17 & 10 & 2 & 94 & Targeted & $11(10-14)$ & Mixture \\
\hline
\end{tabular}

* this is the value in the original sample before exclusions for failed results and no follow up.

Only first author is given for each study. Numbers reported are those after exclusion of cases without a cfDNA test result or pregnancy outcome. Gestational age (GA) is given as median or mean (range). MPSS, massively parallel shotgun sequencing. 
Table 2. Studies reporting on the application of cell-free DNA analysis of maternal blood in screening for trisomy 21.

\begin{tabular}{|c|c|c|c|c|}
\hline \multirow[b]{2}{*}{ Study } & \multicolumn{2}{|r|}{ Trisomy 21} & \multicolumn{2}{|c|}{ Non trisomy 21} \\
\hline & Total & $\begin{array}{l}\text { Detection rate } \\
\text { n }(\%, 95 \% \mathrm{Cl})\end{array}$ & Total & $\begin{array}{c}\text { False positive rate } \\
\text { n }(\%, 95 \% \mathrm{Cl})\end{array}$ \\
\hline Lau $(2013)^{17}$ & 1 & $1(100,25.0-100)$ & 11 & $0(0,0-28.49)$ \\
\hline Huang $(2014)^{18}$ & 9 & $9(100,66.4-100)$ & 180 & $0(0,0-2.03)$ \\
\hline $\operatorname{Tan}(2016)^{19}$ & 4 & $4(100,39.8-100)$ & 506 & $0(0,0-0.73)$ \\
\hline Du $(2017)^{20}$ & 2 & $2(100,15.8-100)$ & 89 & $0(0,0-4.06$ \\
\hline Le Conte $(2018)^{21}$ & 3 & $3(100,29.2-100)$ & 414 & $1(0.24,0.01-1.34)$ \\
\hline Yang $(2018)^{22}$ & 4 & $4(100,39.8-100)$ & 396 & $0(0,0-0.93)$ \\
\hline $\mathrm{Yu}(2019)^{23}$ & 16 & $16(100,79.4-100)$ & 1141 & $0(0,0-0.32)$ \\
\hline Current study & 17 & $16(94.1,71.3-100)$ & 980 & $1(0.10,0-0.57)$ \\
\hline Pooled analysis, $\%,(95 \% \mathrm{Cl})^{*}$ & \multicolumn{2}{|r|}{$98.2(83.2-99.8)$} & \multicolumn{2}{|c|}{$0.05(0.26-0.01)$} \\
\hline Heterogeneity assessment & & 0.020 & & 0.011 \\
\hline Likelihood ratio positive & \multicolumn{4}{|c|}{$1837(369-9149)$} \\
\hline Likelihood ratio negative & \multicolumn{4}{|c|}{$0.018(0.002-0.190)$} \\
\hline
\end{tabular}

*Bivariate random-effects model; $\mathrm{Cl}$, confidence interval. 
Table 3. Studies reporting on the application of cell-free DNA analysis of maternal blood in screening for trisomy 18.

\begin{tabular}{|c|c|c|c|c|}
\hline \multirow[b]{2}{*}{ Study } & \multicolumn{2}{|r|}{ Trisomy 21} & \multicolumn{2}{|c|}{ Non trisomy 21} \\
\hline & Total & $\begin{array}{l}\text { Detection rate } \\
\text { n }(\%, 95 \% \mathrm{Cl})\end{array}$ & Total & $\begin{array}{c}\text { False positive rate } \\
\mathbf{n}(\%, 95 \% \mathrm{Cl})\end{array}$ \\
\hline Huang $(2014)^{18}$ & 2 & $1(50.0,1.3-98.7)$ & 187 & $0(0,0-1.95)$ \\
\hline Le Conte $(2018)^{21}$ & 1 & $1(100,2.5-100)$ & 417 & $0(0,0-0.88)$ \\
\hline Yang $(2018)^{22}$ & 1 & $1(100,2.5-100)$ & 399 & $0(0,0-0.92)$ \\
\hline $\mathrm{Yu}(2019)^{23}$ & 4 & $4(100,39.8-100)$ & 1153 & $0(0,0-0.32)$ \\
\hline Current study & 10 & $9(90,55.5-99.8)$ & 986 & $1(0.10,(0-0.56)$ \\
\hline Pooled analysis, \%, $(95 \% \mathrm{Cl})^{\star}$ & \multicolumn{2}{|c|}{$88.9(64.8-97.2)$} & \multicolumn{2}{|c|}{$0.03 \%(0.33-0.00)$} \\
\hline Heterogeneity assessment & \multicolumn{2}{|r|}{0} & \multicolumn{2}{|r|}{0} \\
\hline Likelihood ratio positive & \multicolumn{4}{|c|}{$2774(388$ - 19823) } \\
\hline Likelihood ratio negative & \multicolumn{4}{|c|}{$0.111(0.30-0.411)$} \\
\hline
\end{tabular}

*Bivariate random-effects model; $\mathrm{Cl}$, confidence interval. 